\title{
L'inspection en Espagne
}

Un gage de qualité

\section{María Antonia Casanova}

Traducteur : Claudine Adam

\section{OpenEdition}

\section{Journals}

Édition électronique

URL : http://journals.openedition.org/ries/3554

DOI : $10.4000 /$ ries. 3554

ISSN : 2261-4265

\section{Éditeur}

Centre international d'études pédagogiques

\section{Édition imprimée}

Date de publication : 1 décembre 1995

Pagination : 113-123

ISSN : 1254-4590

\section{Référence électronique}

María Antonia Casanova «L'inspection en Espagne », Revue internationale d'éducation de Sèvres [En ligne], 08 | 1995, mis en ligne le 01 décembre 1995, consulté le 21 avril 2019. URL : http:// journals.openedition.org/ries/3554; DOI : 10.4000/ries.3554

Ce document a été généré automatiquement le 21 avril 2019

(c) Tous droits réservés 


\title{
L'inspection en Espagne
}

\author{
Un gage de qualité \\ María Antonia Casanova \\ Traduction : Claudine Adam
}

\section{Cadre légal de l'inspection}

1 Pendant les années 1980, le système éducatif espagnol dans son ensemble a subi des changements vastes et importants qui ont touché toutes ses composantes et tous les aspects de son fonctionnement; le même phénomène s'est produit dans la société toute entière, du fait de la transformation de la réalité politique tant sur le plan de la structure de l'État que du mode de gouvernement choisi, c'est-à-dire l'adoption du système démocratique, avec toutes les implications que cela entraîne.

2 Un des axes de fonctionnement du système éducatif se trouve constitué, sans aucun doute, par l'inspection technique de l'Éducation, puisqu'il s'agit du service chargé de contrôler et d'évaluer l'application des normes et leur réelle répercussion dans les établissements scolaires, institutions où, finalement, se concrétisent les faits éducatifs. L'inspection, par conséquent, a été elle aussi concernée ces dernières années par les changements globaux qui ont eu lieu dans la société, et plus précisément, dans l'éducation.

3 L'inspection travaillait selon les normes établies par la Loi générale de l'éducation ${ }^{1}$ et par un décret du 22 mars 1973 qui précisa les missions du service de l'inspection technique de l'éducation. L'inspection était structurée en deux services spécialisés selon les niveaux éducatifs (enseignement primaire et bachillerato); s'y ajoutèrent par la suite, les inspecteurs de formation professionnelle. Cette loi évoquait déjà la possibilité de fusion en un seul corps d'inspection, sans qu'il fût alors donné suite à cette hypothèse.

4 À la suite de la promulgation de la Constitution espagnole de 1978 la structure de l'État subit des changements importants et l'administration éducative commence aussi à se modifier, même si aucune nouvelle Loi générale de l'éducation n'est publiée. Notamment, l'existence, depuis 1981, des communautés autonomes ayant les pleines compétences en 
matières d'éducation, oblige à formuler des normes de base pour l'ensemble de l'État; elles seront développées par ces communautés dans leur domaine territorial. Les fonctionnaires de l'inspection technique de l'éducation sont transférés en même temps que les compétences éducatives ${ }^{2}$, ce qui oblige aussi à créer une haute inspection (sans caractère technique), pour garantir sur la totalité du territoire de l'État le respect par les gouvernements autonomes des normes éducatives de base, et permettre ainsi l'homologation du système dans tout le pays et l'égalité des chances des citoyens, quel que soit l'endroit où ils poursuivent leurs études.

5 En 1984, la structure et l'accès à l'inspection technique de l'éducation sont modifiés ${ }^{3}$ sur les points suivants :

- établissement d'un service unique dans lequel sont intégrés les inspecteurs appartenant aux trois corps correspondant aux niveaux déjà cités. Pour les futurs recrutés, il est prévu de rester dans leur corps d'origine, mais «dans l'exercice de la fonction d'inspecteur de l'éducation »;

- les inspecteurs seront nommés pour trois ans, délai au bout duquel, soit ils devront solliciter la prorogation de leur nomination pour trois années supplémentaires, soit ils pourront réintégrer leur poste antérieur; dans le premier cas ils devront satisfaire aux conditions établies par l'administration éducative, il est également prévu un retour obligatoire à l'enseignement au bout de six années, mais ce dispositif a été supprimé en 1988: les inspecteurs peuvent donc exercer leurs fonctions de façon définitive;

- l'accès à la fonction d'inspecteur se fera en fonction d'une liste d'aptitudes.

Dans la présentation suivante, je me référerai au modèle implanté sur le territoire directement géré par le ministère de l'éducation qui englobe actuellement vingt-sept provincias ${ }^{4}$.

7 Dans les textes législatifs cités, la conception du fonctionnement de l'inspection tient compte, en partie, des nouvelles particularités du système ${ }^{5}$.

8 Les fonctions attribuées à l'inspection sont, sur le fond, les mêmes que celles qu'elle exerçait jusqu'alors, à l'exception de quelques légères différences, prenant en compte les exigences sociales du moment. Par contre, la formation des professeurs, tâche à laquelle l'inspection collaborait avec d'autres institutions de façon remarquable, ne fait plus partie de ses fonctions. Cette formation passe à une nouvelle unité qui apparaît dans chaque direction départementale : l'unité de programmes éducatifs (UPE), chargée, par le biais des centres de professeurs et de ressources, de faire parvenir aux professeurs en exercice l'actualisation et la formation professionnelle nécessaires à un développement efficace de l'enseignement.

9 L'inspection exerce ses fonctions dans le cadre d'un plan général d'action et de plans départementaux d'activités élaborés pour chaque année scolaire, plans qui regroupent les priorités du système pour unifier les actions fondamentales sur tout le territoire.

10 L'inspection s'articule en un Service central et des services provinciales.

11 Les services provinciales d'inspection sont organisés en zones territoriales; chacune est prise en charge par une équipe d'inspecteurs où l'on privilégie l'équilibre entre l'expérience dans les différents niveaux éducatifs et les spécialités académiques de chacun d'entre eux. Certaines tâches pourront être réalisées par tous les inspecteurs, tandis que d'autres seront réservées à celui qui sera le plus qualifié pour les remplir. Chaque zone a un coordinateur. 
12 La formation continue des inspecteurs se déroulera dans le cadre d'un programme qui pourra avoir en tout ou partie un caractère obligatoire.

Les visites dans les établissements scolaires sont répertoriées en plusieurs catégories: habituelles, spécifiques, accidentelles et d'évaluation. Il est conseillé qu'elles soient réalisées par plusieurs inspecteurs.

On définit aussi une série de documents qui homogénéisent le fonctionnement des différents services: le compte rendu de visite, le document de planification hebdomadaire et la fiche de l'établissement.

C'est dans le cadre de cette réglementation que l'inspection de l'éducation a fonctionné au cours de sa dernière étape, après la refonte de 1984. Il y a eu des éléments très positifs, comme le travail en équipe considérablement développé par rapport à la période antérieure. En revanche, d'autres aspects se sont révélés peu efficaces, surtout au début ; par exemple la généralisation excessive des visites effectuées par plusieurs inspecteurs, l'absence d'affectation d'établissements précis à chaque inspecteur pour les questions de fonctionnement habituel ou le fait de ne pas mettre à profit les spécialités ou l'expérience des inspecteurs pour exercer certaines fonctions devenues actuellement absolument nécessaires.

\section{L'inspection centrale}

Placée sous la responsabilité d'un chef de service, l'inspection centrale est chargée des fonctions suivantes :

- suivi des plans provinciales d'activités, et évaluation de leur degré de réalisation ;

- conception, suivi et évaluation du plan d'actualisation et de perfectionnement ;

- inspection des établissements à caractère particulier ${ }^{6}$ dont la charge incombe au service central de l'inspection;

- réalisation de toutes les activités et les visites dont elle sera chargée par délégation du chef de service.

Quelques commentaires pour préciser ces fonctions :

Le plan général d'action est élaboré chaque année par l'inspection centrale. On y trouve les actions prioritaires de toutes les inspections provinciales pour l'année scolaire. À partir de ce plan, chaque Servicio provincial élabore son propre plan, en ajoutant les actions spécifiques nécessaires dans son secteur, et le remet à l'inspection centrale. Au cours de l'année, les inspecteurs centraux visitent les provincias pour effectuer le suivi du plan approuvé. À la fin de chaque année scolaire, un questionnaire est remis à chaque inspecteur provincial responsable d'un secteur, pour autoévaluer l'accomplissement du plan prévu. Lorsque les questionnaires remplis ont été reçus par l'inspection centrale, on élabore avec l'ensemble des résultats d'auto-évaluation des services provinciales et du service central un mémoire qui est publié et transmis aux autorités ministérielles.

Tous les trois ans, un plan d'actualisation et de perfectionnement des inspecteurs est élaboré, à partir d'un questionnaire destiné à détecter les besoins de formation et à établir les attentes du système éducatif par rapport à l'inspection. C'est le deuxième plan qui est actuellement en application; il se terminera en 1996. Il comporte des cours, centralisés ou décentralisés, des groupes de travail, des séminaires, des congrès ou journées diverses, etc. On peut donner un chiffre significatif sur la réalisation du premier 
plan : quarante-trois cours ont eu lieu auxquels ont assisté plus de deux mille trois cents personnes.

Il est important également de signaler qu'au début de cette action on a commencé à envoyer une bibliographie actualisée aux inspections (centrale et provinciales), dans le but de créer ou d'enrichir et d'actualiser les bibliothèques, existantes ou non à ce moment-là. Étant donné que l'on commençait à appliquer une nouvelle loi, cette mesure est apparue comme un élément de base indispensable au suivi de la formation dispensée dans les cours, en rapport avec les nouveautés enregistrées dans l'enseignement actuel, en matière d'épistémologie, d'organisation et de didactique.

21 À la fin de chaque plan d'actualisation, un mémoire est élaboré, résumant les actions réalisées et évaluant les résultats obtenus.

Pour mener à bien ces tâches, l'inspection centrale s'est organisée en équipes (dont le nombre peut varier) qui prennent en charge certaines actions, bien que presque tous les inspecteurs centraux y participent. C'est ainsi qu'actuellement, il existe six équipes chargées de la coordination des services provinciales d'inspection; de l'actualisation et du perfectionnement des inspecteurs de l'éducation; de l'évaluation; des statistiques; du suivi des établissements à caractère particulier et du statut de l'inspection. Pour les travaux d'évaluation elle a élaboré en collaboration avec le $\mathrm{CIDE}^{7}$, le plan d'évaluation des établissements, actuellement appliqué dans les établissements publics dépendant du ministère. Elle a réalisé également des études statistiques variées sur les résultats globaux obtenus par les élèves dans les différents niveaux éducatifs ou dans les différentes disciplines, etc. Enfin, l'équipe chargée du statut du corps étudie tout ce qui concerne l'accès, les barèmes ou l'organisation interne de l'inspection ${ }^{8}$.

D'une part, la contribution fondamentale de l'inspection centrale au fonctionnement global de l'ensemble de l'inspection sur le territoire dépendant du ministère consiste à coordonner et harmoniser ses actions et ses critères, afin que, outre les activités qui doivent être réalisées habituellement ou les questions spécifiques à chaque provincia, la politique ministérielle puisse indiquer les priorités de chaque année scolaire et savoir qu'elles vont être contrôlées et soutenues sur tout le territoire; tout particulièrement durant les premières années d'implantation d'une nouvelle loi et d'une nouvelle structure du système éducatif, en raison de la complexité et des risques que cela entraîne. Cette intervention permet aussi d'obtenir des données unifiées et plus facilement évaluables, ce dont on ne peut dénier l'importance. D'autre part, le perfectionnement des inspecteurs dans le cadre d'un plan permet de garantir qu'ils reçoivent toutes les informations de base nécessaires à leurs actions ainsi que l'actualisation dont ils ont besoin pour aborder leur profession avec des garanties techniques.

Après cette évaluation positive du rôle joué par l'inspection centrale dans l'ensemble du système éducatif, il convient de noter que, dès que toutes les communautés autonomes espagnoles disposeront des pleines compétences en matière d'éducation, ce rôle de coordination ou de regroupement d'activités aujourd'hui réalisé par l'inspection centrale disparaîtra, comme c'est le cas dans les communautés qui les exercent déjà. C'est pourquoi, l'administration doit réorganiser sa structure pour que cette inspection exerce son travail de contrôle des établissements, en plus d'autres possibilités de coordination des Hautes inspections existant dans les communautés autonomes. 


\section{Les fonctions de l'inspection}

L'exercice des fonctions d'inspection, évoquées précédemment, doit influer de façon décisive sur l'amélioration de la qualité du système éducatif, puisqu'elles visent à contrôler, guider et évaluer les projets et les résultats obtenus, aussi bien au terme d'un temps déterminé que pendant leur processus de fonctionnement. Cela doit permettre, en retour, de réalimenter constamment le système pour l'améliorer de façon continue et aider l'administration à prendre des décisions pertinentes, puisqu'elle peut obtenir des données fiables sur la réalité des institutions scolaires.

Analysons chacune des fonctions dont est chargée l'inspection de l'éducation, selon leur importance vis-à-vis de la qualité du système.

Veiller au respect des lois, règlements et autres dispositions établis par l'administration de l'éducation dans les établissements d'enseignement et les services, ainsi qu'à l'exécution et au déroulement des programmes et activités à caractère éducatif approuvés ou autorisés par le ministère de l'éducation.

Surveiller ou contrôler la mise en œuvre des normes légales est une des fonctions de base de l'inspection, ce fut même en Espagne le motif de sa création. C'est la première et la seule dont elle ait été chargée depuis une époque antérieure à Philippe II et qui se soit maintenue sans interruption tout au long des années. L'inspection ayant sa raison d'être dans la garantie d'une éducation de qualité pour tous les citoyens (enfants et jeunes) et l'État disposant, par des règlements, des mécanismes pour y aboutir, contrôler le fonctionnement correct de ces normes, c'est garantir l'accès de tous, en particulier ceux dont le niveau culturel est le plus bas, à ce qui est mise en place. Le rôle de l'inspection est donc crucial pour garantir au citoyen le droit à l'éducation reconnu par la Constitution espagnole. D'autre part, dans des périodes de réforme éducative vaste et profonde, l'administration a besoin de connaître, presqu'au jour le jour, l'incidence de chaque norme sur les établissements, les difficultés qui émanent de son application, les effets positifs qui en découlent... Cette information, seule l'inspection peut la fournir, car elle a des attributions suffisantes pour recueillir directement et rigoureusement les données et les faire parvenir aux autorités correspondantes.

Collaborer aux réformes éducatives, aux activités de perfectionnement des professeurs, au processus de rénovation pédagogique et aux programmes expérimentaux, ainsi que participer à leur diffusion, suivi et évaluation.

La définition de cette fonction semble en désaccord avec ce qui a été dit sur l'absence de participation de l'inspection à la formation des professeurs. Dans les faits, ce sont l'unité de programmes éducatifs et les centres de professeurs qui assument le perfectionnement des professeurs en exercice. Collaborer signifie donc ici pour l'inspection accomplir le reste des fonctions dont elle est chargée ; cela s'identifie presque à sa tâche de conseil, car cela peut consister en une information ponctuelle sur des sujets précis, mais sans constituer pour autant une formation systématique. Le reste de l'énoncé se traduit différemment en fonction des moments. Selon les besoins du système, on charge l'inspection de tâches spécifiques pour diffuser et évaluer les innovations ou les processus de formation concrets. visite les établissements, détermine les besoins de formation des professeurs de sa zone et 
informe le directeur provincial. Celui-ci en informe l'unité de programmes, qui doit mettre en place les mécanismes permettant à ces professeurs d'avoir accès aux cours, groupes de travail, conférences, etc., qui seront nécessaires. Mais pour que ce schéma fonctionne efficacement, une coordination étroite et souple entre inspection et unité de programmes s'avère indispensable; sinon, entre la bureaucratie et la lenteur dans l'adoption des décisions, on perd toute efficacité. C'est pour cette raison, entre autres, que le projet ainsi décrit a été - et continue à être - une source permanente de difficultés et de problèmes au sein des administrations provinciales ainsi que dans les établissements en fonction de leur manière d'agir. Les inspecteurs étant qualifiés sur les plans scientifique, organisationnel et didactique, les chefs d'établissement et les professeurs se tournent vers eux pour résoudre les problèmes auxquels ils se trouvent confrontés au quotidien dans leur travail, sans attendre l'arrivée du «formateur ». Le problème reste brûlant, car les établissements font savoir qu'ils reçoivent peu d'aide et, paradoxalement, ils ne la réclament pas aux personnes chargées de la leur apporter. Manque de confiance envers les «formateurs »? Manque de la préparation requise chez ces derniers? Protester pour protester? Les raisons sont diverses. On cherche des solutions et il faut espérer que l'on trouvera la mieux adaptée.

Il faut ajouter, en dernier lieu, que l'inspection collabore très activement, aux nombreux cours organisés dans les centres de professeurs, en intervenant, à titre individuel et à la demande des organisateurs, chacun selon sa spécialité.

Évaluer le rendement du système éducatif, par l'analyse de l'organisation, du fonctionnement et des résultats des établissements d'enseignement et des services, et par l'analyse de l'exécution et du déroulement des programmes et activités à caractère éducatif lancés ou autorisés par le ministère de l'éducation.

L'évaluation et le contrôle sont deux tâches spécifiques et prioritaires de l'inspection de l'éducation. Ces deux fonctions sont solidaires et complémentaires. En outre, c'est dans la fonction d'évaluation que réside l'amélioration de la qualité du système éducatif, tout spécialement lorsqu'on l'exerce dans un sens éminemment formateur, plutôt que sommatif ou classificateur. Il est presque possible d'affirmer que l'objectif de l'évaluation formative et celui de l'expertise globale coïncident. Si l'objectif de l'inspection est d'optimiser le rendement du système et celui de l'évaluation formative de perfectionner les processus éducatifs, tous deux se rejoignent dans une finalité commune: l'amélioration de la qualité du système éducatif dans son ensemble. C'est ainsi que l'évaluation formative devient un instrument indispensable pour le travail de l'inspecteur.

Une évaluation formative des processus de fonctionnement des établissements associée à une évaluation des résultats obtenus fait de l'expertise une pièce clé pour améliorer la qualité de l'enseignement d'un pays. Cette mission pourrait certes, être réalisée par d'autres instances, mais ce nouveau groupe de personnes, sous un autre nom, deviendrait finalement une « inspection parallèle », puisque pour obtenir les données que possède l'inspection il devrait disposer des fonctions et attributions que celle-ci possède dans l'organigramme général du système éducatif.

36 Pour en venir au travail pratique d'évaluation réalisé par l'inspection, en plus de son évaluation permanente des établissements grâce aux visites systématiques et aux rapports et propositions qui en découlent, on applique depuis maintenant cinq années scolaires, un plan d'évaluation des établissements, accepté des différents secteurs de la communauté éducative et jugé favorablement jusqu’à présent en raison des effets 
produits dans les établissements évalués. Il faut aussi souligner en ce moment, l'importance de l'évaluation de l'application des projets éducatif et curriculaire dans les établissements, puisqu'elle est effectuée systématiquement pour la première fois dans notre système éducatif ; l'estimation adéquate qui en sera faite sera essentielle pour lui permettre d'atteindre toute son efficacité ou pour décider de son inutilité dans l'organisation de la structure des diverses institutions éducatives.

Conseiller et informer les différents secteurs de la communauté éducative et les organes individuels et collégiaux des établissements d'enseignement dans le cadre des compétences et des attributions dont chacun d'eux est chargé par la réglementation en vigueur.

La tâche de conseil et d'information est, elle aussi, inhérente à l'inspection de l'éducation. Dans certains pays européens et américains, c'est même là sa tâche prioritaire et principale. En général, les conseils que donne l'inspection espagnole concernent davantage les aspects de la loi, de l'organisation et de l'évaluation que ceux de type purement didactique. Dans ce cas, son rôle ne se recoupe avec aucune autre entité du système.

Il est pourtant fréquent que cette fonction soit partagée avec d'autres instances et qu'elle soit, par conséquent, source de conflits quand les tâches des uns et des autres ne sont pas clairement définies. Établir distinctement la limite de l'intervention de chaque organisme dans l'établissement d'enseignement est fondamental pour l'organisation de ce dernier.

L'appui permanent, fruit de la relation établie lors des visites habituelles des établissements, constitue un élément décisif pour guider l'innovation, et par conséquent sa progression vers la qualité de fonctionnement souhaitée?.

Collaborer avec l'unité de planification pour étudier les besoins éducatifs de chaque province. Informer sur tout ce que lui aura demandé l'autorité compétente ou ce qui aura été porté à sa connaissance dans l'exercice de son activité.

Dans les deux cas, les propositions adéquates, à supposer qu'il y en eût, seront transmises par les voies réglementaires.

Ces fonctions regroupent d'autres actions parmi celles, si nombreuses, menées à bien par l'inspection, et il est pratiquement impossible de les détailler en raison de leur ampleur, de leur nombre et des domaines qu'elles englobent. D'autre part, l'inspection donne en permanence des informations sur tout ce qu'elle considère intéressant pour la prise de décisions de l'administration et sur tout ce qui est important pour l'amélioration de l'établissement.

\section{Inspection de qualité : qualité du système éducatif ?}

Il faut en convenir, une institution éducative telle que l'inspection doit avoir une raison d'être particulièrement bonne pour pouvoir durer - avec des caractéristiques différentes selon les époques - durant six siècles, du moins pour ce qui est de l'Espagne, le premier document écrit où elle apparaît datant de 1370 . Sur quels traits génériques peut se baser le maintien de l'inspection? On les trouvera probablement dans ces trois raisons :

- le besoin qu'a toujours manifesté l'État de connaître la situation réelle de l'application des lois qu'il promulgue (fonction de contrôle);

- l'évaluation du fonctionnement des établissements régis par ces lois, en vue de leur amélioration continuelle (fonction d'évaluation) ; 
- enfin, la nécessité d'amélioration de la qualité d'éducation qu'un pays offre à ses citoyens et de garantir leurs droits par rapport à cet enseignement établi légalement. réunions où se retrouvent périodiquement les inspections centrales ou générales de l'Union européenne: il serait indispensable de créer une association des inspections européennes (générales, centrales, régionales, provinciales, ...) : l'important n'est pas l'intitulé, mais la fonction susceptible de contribuer à l'articulation des systèmes éducatifs européens qui sont appelés à trouver des objectifs communs pour l'ensemble 
des citoyens de cette nouvelle entité. Je crois qu'il faut y arriver pour garantir un fonctionnement adapté et équivalent de l'enseignement en Europe. Sans compter l'enrichissement personnel et professionnel que l'on retire toujours d'un échange avec des collègues et qui serait, à lui seul, une raison suffisante pour mettre en œuvre l'association des inspecteurs européens.

\section{NOTES}

1. Loi générale de l'éducation et du financement de la réforme éducative, 4 août 1970, article 142 et 143 .

2. Sur l'inspection dans les communautés autonomes, voir, dans ce numéro, l'article de Manuel Narbona.

3. Loi du 2 août 1984 concernant les mesures pour la réforme de la fonction publique.

4. Chaque région espagnole est constituée de plusieurs provincias, entités administratives et territoriales souvent comparées aux départements français. Rappelons que, parmi les dix-sept régions espagnoles, celles qui n'ont pas encore le statut de communauté autonome sont gérées par l'administration centrale (NDLR).

5. Comme la structure actuelle du système éducatif réglementé par la LOGSE n'est pas encore implantée, seules ont pu être considérées quelques-unes de ses caractéristiques ; les besoins réels du système sont vérifiés en ce moment, au fur et à mesure que se généralisent d'année en année les étapes prévues. L'activité des inspecteurs est ajustée en fonction de ces besoins, bien que les normes légales qui les régissent ne soient pas modifiées. Sur ce dernier point, deux textes de référence : le décret royal du 15 décembre 1389, qui définit les fonctions et l'organisation du service de l'inspection technique de l'éducation, et l'ordre ministériel du 27 septembre 1530, qui réglemente le fonctionnement de ce service.

6. On désigne ainsi les établissements, sections ou organismes culturels du système éducatif espagnol implantés à l'étranger. La participation à l'inspection des écoles européennes est généralement comprise dans ce cadre.

7. Centre de recherches, documentation et évaluation, au moment où ce travail était réalisé ; à l'heure actuelle, la fonction d'évaluation de cet organisme est exercée, avec d'autres caractéristiques, par l'Institut national de la qualité et de l'évaluation.

8. L'inspection effectue aussi une multitude de travaux additionnels qui lui sont demandés chaque année par différentes instances ou qui sont devenus une habitude de la fonction. Par exemple, la participation aux commissions de sélection des professeurs nommés dans les établissements étrangers, ou l'organisation et le déroulement du cours d'« expertise globale du système éducatif »- dont le niveau professionnel est très élevé et qui a lieu chaque année dans le cadre du programme de coopération avec l'Amérique ibérique.

9. Un exemple suffira: il est tout à fait crucial d'élaborer le projet éducatif et le projet curriculaire, leur aménagement interne, etc., de façon correcte pour pouvoir faire en sorte que leur mise en œuvre soit efficace. Dans cette perspective, l'inspection s'est révélée un appui indiscutable pour mener à bien ces documents institutionnels. 


\section{RÉSUMÉS}

La réforme du système éducatif a eu des incidences partielles sur le statut, le recrutement, les tâches confiées aux inspecteurs ainsi que l'organisation territoriale de l'inspection. L'inspection centrale veille à garantir la qualité du système éducatif en assurant la coordination des inspections provinciales pour les Communautés qui ne disposent pas encore des pleines compétences en matière d'éducation.

INDEX

Index géographique : Espagne

Mots-clés : inspecteur, inspection, système éducatif

\section{AUTEURS}

\section{MARÍA ANTONIA CASANOVA}

Inspecteur central de l'éducation, ministère de l'éducation, Madrid, Espagne. 